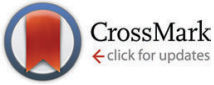

Cite this: Chem. Commun., 2015, 51, 10624

Received 28th April 2015, Accepted 29th May 2015

DOI: $10.1039 / \mathrm{c} 5 \mathrm{cc} 03557 \mathrm{k}$

www.rsc.org/chemcomm

\section{Functional native disulfide bridging enables delivery of a potent, stable and targeted antibody-drug conjugate (ADC) $\dagger$}

\author{
João P. M. Nunes, ${ }^{a}$ Maurício Morais, ${ }^{a}$ Vessela Vassileva, ${ }^{b}$ Eifion Robinson, ${ }^{a}$ \\ Vineeth S. Rajkumar, ${ }^{\text {b }}$ Mark E. B. Smith, ${ }^{a}$ R. Barbara Pedley, ${ }^{b}$ Stephen Caddick, ${ }^{a}$ \\ James R. Baker*a and Vijay Chudasama*a
}

\begin{abstract}
Herein we report the use of next generation maleimides (NGMs) for the construction of a potent antibody-drug conjugate (ADC) via functional disulfide bridging. The linker has excellent stability in blood serum and the ADC, armed with monomethyl auristatin $E$ (MMAE), shows excellent potency and cancer cell selectivity in vitro.
\end{abstract}

Antibody-drug conjugates (ADCs) are comprised of antibodies that are armed with warheads using appropriate linker technologies. ${ }^{1,2}$ They combine the exquisite targeting ability of antibodies (i.e. allowing for discrimination between healthy and diseased tissue) with the cell-killing ability of cytotoxic drugs. This class of targeted therapy has shown considerable promise in the treatment of various cancers in recent years with two FDA-approved ADCs coming onto the market recently (i.e. Adcetris ${ }^{\mathrm{TM}}$ and Kadcyla $^{\mathrm{TM}}$ ) and with over $30 \mathrm{ADCs}$ in the clinic. ${ }^{3}$ However, in order for ADCs to deliver their full potential, sophisticated conjugation technologies to connect the warhead to the antibody are vital. ${ }^{4}$ Conjugation to antibodies is typically achieved through either multiple lysine modification or by functionalisation of thiols generated by reduction of interchain disulfide bonds; neither of these is ideal (Fig. 1). ${ }^{4}$ Lysine modification is sub-optimal as it generates heterogeneous ADCs, which have been shown to have a narrow therapeutic window relative to homogeneous ADCs. ${ }^{5}$ Cysteine modification, following interchain disulfide reduction, results in the permanent loss of structural disulfide bonds, which may reduce the stability of the ADC in vivo. ${ }^{4}$ It also generates heterogeneous mixtures when targeting typical drug-to-antibody ratios (DARs) of 2-4. Other approaches using cysteine-based sitedirected mutagenesis and unnatural amino acids should also be highlighted. ${ }^{5,6}$ However they also have limitations, e.g. disulfide

\footnotetext{
${ }^{a}$ Department of Chemistry, University College London, London, WC1H OAJ, UK. E-mail:j.r.baker@ucl.ac.uk,v.chudasama@ucl.ac.uk; Tel: +44 (0)20 7679 2653, $+44(0) 2076792077$

${ }^{b}$ UCL Cancer Institute, 72 Huntley Street, London, UK

$\dagger$ Electronic supplementary information (ESI) available: ${ }^{1} \mathrm{H}$ and ${ }^{13} \mathrm{C}$ NMR spectra for all small molecules, SDS-PAGE gels for bioconjugates. See DOI: 10.1039/ c5cc03557k
}
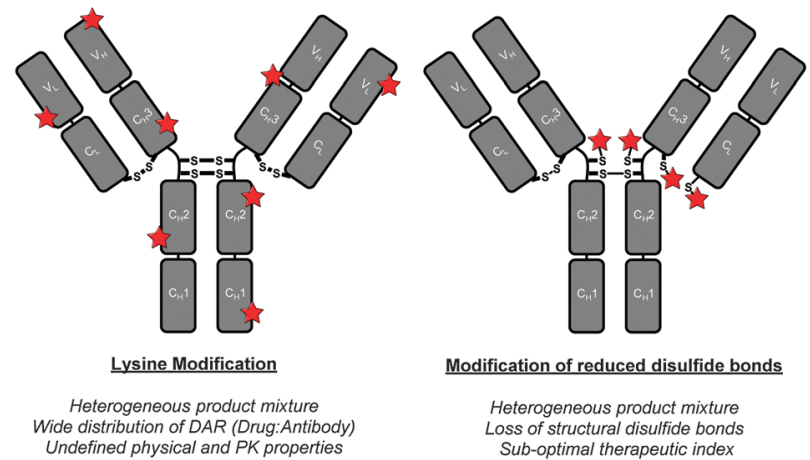

Modification of reduced disulfide bonds

Heterogeneous product mixture Loss of structural disulfide bonds Sub-optimal therapeutic index

Fig. 1 Classical approaches to ADC construction.

scrambling post-reduction and high cost combined with relatively low expression yields, respectively.

Recently, we have described methods for the insertion of next generation maleimides (NGMs) into disulfide bonds in various proteins, engineered antibody single-chain variable fragments ( $\mathrm{ScFv}$ ), a fragment antigen-binding (Fab) construct and a full antibody to yield site-selectively modified NGM conjugates (Scheme 1). ${ }^{7}$ The NGM conjugate may be hydrolysed to a maleamic acid. ${ }^{7 b}$ Having optimised the chemistry to modify proteins with NGMs, herein we describe the construction of an industry relevant $\mathrm{ADC}$ (i.e. an antibody functionalised with a highly potent drug) and evaluate its biological relevance in terms of stability in blood serum, and efficacy plus cancer cell selectivity in vitro.

Initially, we had to select a suitable full antibody platform to appraise the application of our technology. Trastuzumab

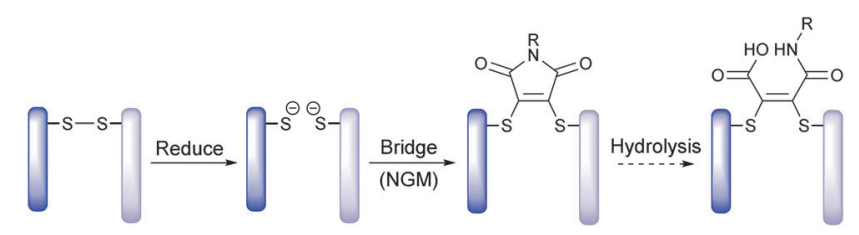

Scheme 1 Exemplification of NGM technology for insertion into a disulfide bond and optional hydrolysis. 
(Herceptin $^{\mathrm{TM}}$ ) is a monoclonal IgG1 antibody that targets the internalising HER2 receptor and has been used successfully in the treatment of HER2+ breast cancer. ${ }^{8}$ Furthermore, it is the antibody component of trastuzumab emtansine (Kadcyla ${ }^{\mathrm{TM}}$ ), a recently FDA-approved ADC therapy for the same indication. ${ }^{9}$ We therefore chose trastuzumab as the full antibody targeting system on which to evaluate this research. We also needed to select a suitable warhead for this study and decided to use anticancer drug monomethyl auristatin E (MMAE) as its suitability as the drug component of ADCs is well-established. ${ }^{3,10}$ Finally, we needed to select a suitable fluorophore to appraise the blood serum stability of the NGM linkage. To this end, we selected Alexa Fluor ${ }^{\circledR} 488$ owing to its established use in this context. ${ }^{10 b}$

Our study began by testing the blood serum stability of NGMtrastuzumab antibody conjugates against a classical maleimide analogue. It has been reported that classical maleimide conjugates are unstable in serum, due to their propensity to undergo retroconjugate additions, leading to transfer of the attached cargo onto blood thiols; particularly albumin. ${ }^{11}$ It is vital in the next generation of ADCs that this vulnerability is avoided, i.e. to limit off-target toxicity associated with premature drug release or drug transfer. Whilst spontaneous retro-conjugate additions are not mechanistically possible in NGM conjugates, cleavage by a thiol addition-elimination mechanism is. ${ }^{7 f}$ However, this mode of reactivity can be precluded by hydrolysis to maleamic acids, which are unreactive to thiols. ${ }^{7 b}$ To test whether NGM conjugates (non-hydrolysed and hydrolysed) offer more suitable platforms for serum stability than classical maleimides we constructed fluorescent trastuzumab antibody conjugates 1-3. This was achieved by conjugation of reagents $\mathbf{4}$ and $\mathbf{5}$ to reduced trastuzumab and carrying out CuAAC click attachments of Alexa Fluor $488^{\circledR}$ azide. This afforded conjugates 1 and 2. It was notable that conjugate 2 was obtained with a fluorophore-to-antibody ratio of 3.91, consistent with conjugation of an NGM reagent to each of the four accessible interchain disulfide bonds. Conjugate 3 was obtained by hydrolysis of conjugate 2 , and retained a fluorophore loading of ca. 4 (see $\mathrm{ESI} \dagger$ for further details).

We appraised the blood serum stability of conjugates 1-3 by analysing fluorescence (post-HPLC separation) of antibody, albumin and "free" fluorophore as a function of days of incubation (Fig. 2, see ESI $\dagger$ for further details). As expected, the study with classical maleimide conjugate 1 revealed substantial transfer of the fluorophore to albumin after 4 days. Interestingly, dithiomaleimidederived conjugate 2 gave a similar profile, suggesting that, albeit via a different mechanism, thiol exchange was occurring with albumin's cysteine 34 at a similar rate. In sharp contrast, maleamic acid conjugate 3 was completely stable, even over a prolonged period of 7 days. From this data it can be concluded that the optimum NGMADC platform would incorporate a maleamic acid linker to ensure robust serum stability.

Following on from the successful blood serum stability experiments with a maleamic acid linker, we synthesised NGM-MMAE construct 6 for direct conjugation onto trastuzumab (Fig. 3). To do this, dithiomaleimide acid 7 was coupled to aminePEG12-tert-butylester, followed by TFA deprotection to afford dithiomaleimide-PEG12-acid 8. This was then coupled to MMAE to form NGM-MMAE 6.
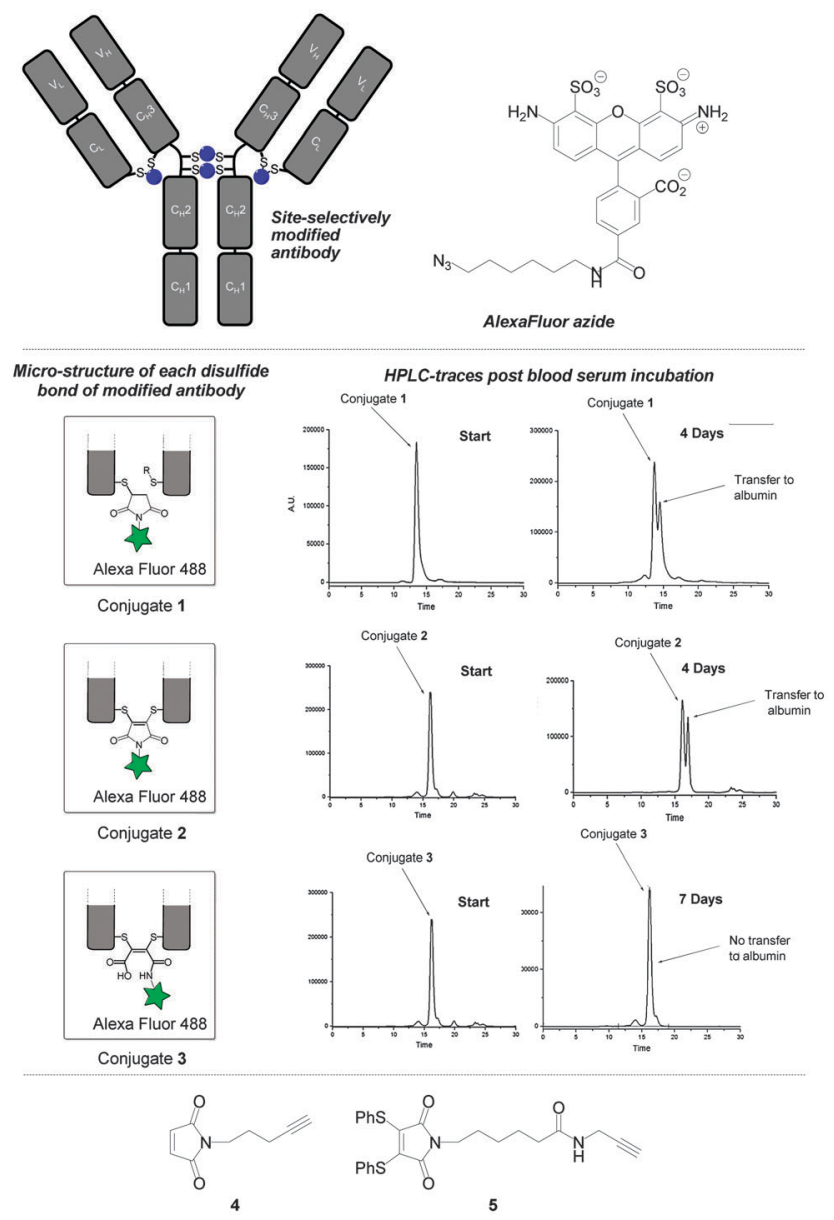

Fig. 2 Blood serum stability of conjugates 1-3 and structure of thiol reactive reagents 4 and 5 .

With NGM-MMAE 6 in-hand, we applied our optimised conditions for the insertion of NGMs into the full antibody system of trastuzumab with the aim of functionally re-bridging its native interchain disulfide bonds. To do this, we only used a small excess of reducing agent (i.e. TCEP, 6 eq.) and NGM-MMAE (5 eq.) to ensure complete reaction and to target a drug-to-antibody ratio (DAR) of $c a$. 4. This was followed by hydrolysis at $\mathrm{pH} 8.4$ at $20{ }^{\circ} \mathrm{C}$ for $72 \mathrm{~h}$ to form thiol-stable NGM-MMAE-ADC 9. This gave an excellent average DAR of 3.89 by HIC analysis (Fig. 4, see ESI $\dagger$ for details on analysis), which is consistent with our Alexa Fluor ${ }^{\circledR} 488$ loading. The ADC therapeutic was formed in good yield and in an efficient manner with retention of binding confirmed by ELISA analysis (see ESI $\dagger$ for details).

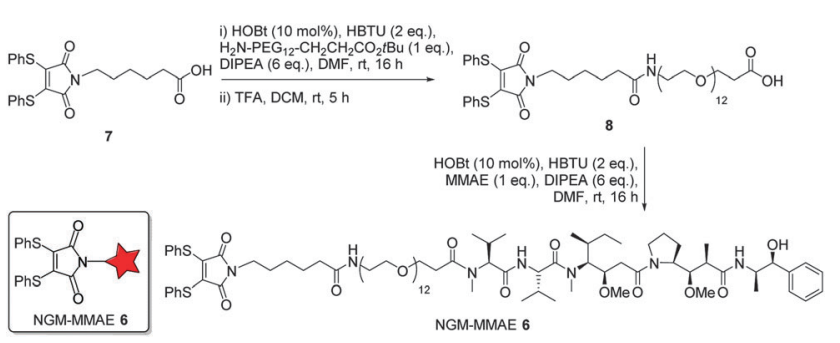

Fig. 3 Synthesis of MMAE-NGM construct 6 . 

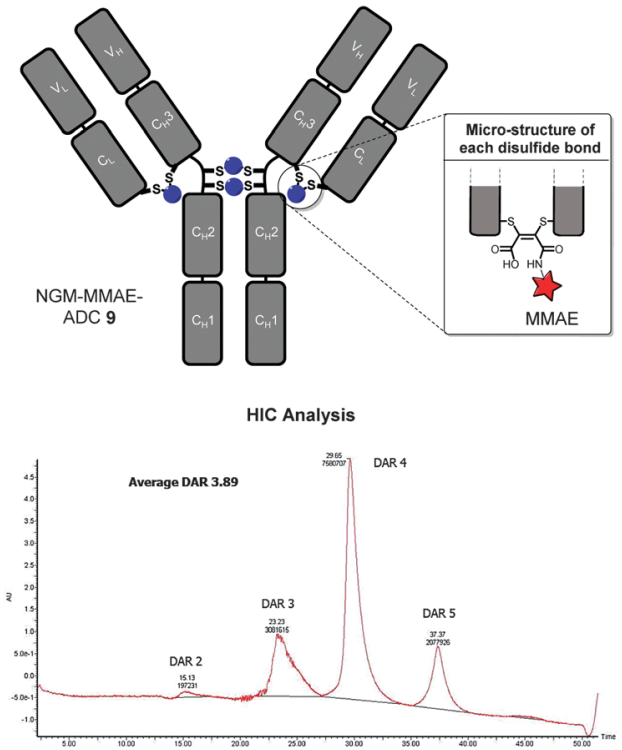

Fig. 4 NGM-MMAE-ADC 9 with HIC analysis.

With an industry relevant ADC in-hand, we appraised its effectiveness in vitro. To appraise the selectivity and potency of the constructs we used breast cancer cell lines SKBR-3 (HER2-positive) and MCF-7 (HER2-negative). As a comparable reduction in cell viability is observed for both cell lines at the same concentration of MMAE ( $\mathrm{IC}_{50}=0.23 \mathrm{nM}$ and $0.50 \mathrm{nM}$ for SKBR-3 and MCF-7 respectively), ${ }^{10 b}$ the cell lines are appropriate for the study, thus paving the way for cytotoxicity studies with NGM-MMAE-ADC 9 (Fig. 5). Gratifyingly, SKBR-3 cell viability was reduced significantly when incubated with the conjugate $\left(\mathrm{IC}_{50}=0.33 \mathrm{nM}\right)$, especially when compared to the control of native Herceptin where the reduction in cell survival was minimal at relatively high concentrations. Additionally, highlighting the targeted delivery aspect of the work, no toxicity was observed in analogous studies with MCF-7 cells incubated with NGM-MMAE-ADC 9. These results clearly highlight the selectivity of our conjugate over MMAE alone, and

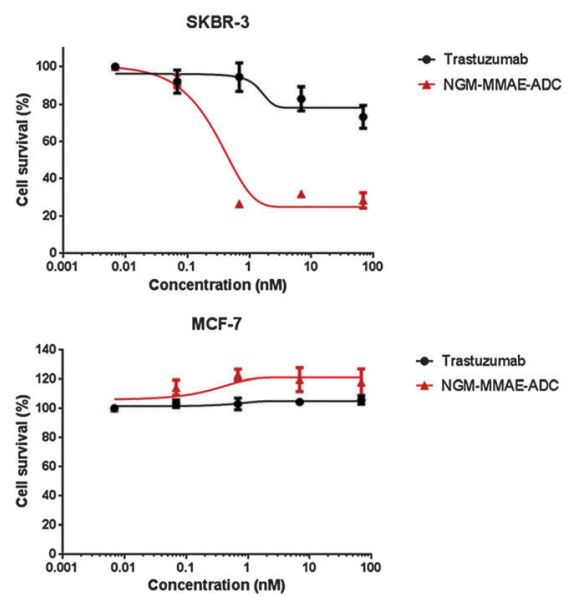

Fig. 5 Inhibition of cell proliferation in cancer cell lines SKBR-3 (HER2positive) and MCF-7 (HER2-negative) for NGM-MMAE-ADC 9 with trastuzumab control. The error bars are the standard error of the mean. indicate that the toxic drug in our conjugates is delivered by an HER2-dependent internalisation mechanism.

In conclusion, NGM linkages have been shown to functionally re-bridge the native disulfide bonds of a full antibody, to be stable in blood serum post-hydrolysis and successfully used for the preparation of an industry relevant NGM-MMAE-ADC. The NGM-MMAE-ADC was shown to selectively target and kill HER2+ cells, thus highlighting the potential of our chemistry for the production of efficacious ADCs. We will next seek to evaluate NGM-ADCs in an in vivo setting, alongside our antibody fragment and antibody bispecific platforms.

The authors gratefully acknowledge the EPSRC, BBSRC, BRC, Wellcome Trust, HEFCE, SBC, MRC, UCL and UCLB for support of our programme. Dr Rajkumar is funded by the CCIC collaboration -CRUK \& EPSRC Comprehensive Cancer Imaging Centre at KCL \& UCL, which is jointly funded by Cancer Research UK and the Engineering and Physical Sciences Research Council (EPSRC).

\section{Notes and references}

1 (a) P. Sapra, A. T. Hooper, C. J. O'Donnell and H.-P. Gerber, Expert Opin. Invest. Drugs, 2011, 20, 1131; (b) S. C. Alley, N. M. Okeley and P. D. Senter, Curr. Opin. Chem. Biol., 2010, 14, 529.

2 J. R. Adair, P. W. Howard, J. A. Hartley, D. G. Williams and K. A. Chester, Expert Opin. Biol. Ther., 2012, 12, 1191.

3 A. Mullard, Nat. Rev. Drug Discovery, 2013, 12, 329.

4 (a) J. A. Flygare, T. H. Pillow and P. Aristoff, Chem. Biol. Drug Des., 2013, 81, 113; (b) L. Ducry and B. Stump, Bioconjugate Chem., 2010, $21,5$.

5 (a) J. R. Junutula, K. M. Flagella, R. A. Graham, K. L. Parsons, E. Ha, H. Raab, S. Bhakta, T. Nguyen, D. L. Dugger, G. Li, E. Mai, G. D. Lewis Phillips, H. Hiraragi, R. N. Fuji, J. Tibbitts, R. Vandlen, S. D. Spencer, R. H. Scheller, P. Polakis and M. X. Sliwkowski, Clin. Cancer Res., 2010, 16, 4769; (b) J. R. Junutula, H. Raab, S. Clark, S. Bhakta, D. D. Leipold, S. Weir, Y. Chen, M. Simpson, S. Ping Tsai, M. S. Dennis, Y. Lu, Y. G. Meng, C. Ng, J. Yang, C. C. Lee, E. Duenas, J. Gorrell, V. Katta, A. Kim, K. McDorman, K. Flagella, R. Venook, S. Ross, S. D. Spencer, W. Lee Wong, H. B. Lowman, R. Vandlen, M. X. Sliwkowski, R. H. Scheller, P. Polakis and W. Mallet, Nat. Biotechnol., 2008, 26, 925.

6 E. S. Zimmerman, T. H. Heibeck, A. Gill, X. Li, C. J. Murray, M. R. Madlansacay, C. Tran, N. T. Uter, G. Yin, P. J. Rivers, A. Y. Yam, W. D. Wang, A. R. Steiner, S. U. Bajad, K. Penta, W. Yang, T. J. Hallam, C. D. Thanos and A. K. Sato, Bioconjugate Chem., 2014, 25, 351.

7 (a) F. F. Schumacher, V. A. Sanchania, B. Tolner, Z. V. F. Wright, C. P. Ryan, M. E. B. Smith, J. M. Ward, S. Caddick, C. W. M. Kay, G. Aeppli, K. A. Chester and J. R. Baker, Sci. Rep., 2013, 3, 1525; (b) C. P. Ryan, M. E. B. Smith, F. F. Schumacher, D. Grohmann, D. Papaioannou, G. Waksman, F. Werner, J. R. Baker and S. Caddick, Chem. Commun., 2011, 47, 5452; (c) F. F. Schumacher, M. Nobles, C. P. Ryan, M. E. B. Smith, A. Tinker, S. Caddick and J. R. Baker, Bioconjugate Chem., 2011, 22, 132; (d) M. E. B. Smith, F. F. Schumacher, C. P. Ryan, L. M. Tedaldi, D. Papaioannou, G. Waksman, S. Caddick and J. R. Baker, J. Am. Chem. Soc., 2010, 132, 1960; (e) L. Castañeda, A. Maruani, F. F. Schumacher, E. Miranda, V. Chudasama, K. A. Chester, J. R. Baker, M. E. B. Smith and S. Caddick, Chem. Commun., 2013, 49, 8187; $(f)$ V. Chudasama, M. E. B. Smith, F. F. Schumacher, D. Papaioannou, G. Waksman, J. R. Baker and S. Caddick, Chem. Commun., 2011, 47, 8781; $(g)$ F. Bryden, A. Maruani, H. Savoie, V. Chudasama, M. E. B. Smith, S. Caddick and R. W. Boyle, Bioconjugate Chem., 2014, 25, 611; (h) F. F. Schumacher, J. P. M. Nunes, A. Maruani, V. Chudasama, M. E. B. Smith, K. A. Chester, J. R. Baker and S. Caddick, Org. Biomol. Chem., 2014, 12, 7261; (i) A. Maruani, S. Alom, P. Canavelli, M. T. W. Lee, R. E. Morgan, V. Chudasama and S. Caddick, Chem. Commun., 2015, 51, 5279; (j) A. Maruani, M. E. B. Smith, E. Miranda, K. A. Chester, V. Chudasama and S. Caddick, Nat. Commun., 2015, 6, 6645.

8 C. A. Hudis, N. Engl. J. Med., 2007, 357, 39. 
9 S. Verma, D. Miles, L. Gianni, I. E. Krop, M. Welslau, J. Baselga, M. Pegram, D.-Y. Oh, V. Diéras, E. Guardino, L. Fang, M. W. Lu, S. Olsen and K. Blackwell, N. Engl. J. Med., 2012, 367, 1783.

10 (a) S. O. Doronina, B. E. Toki, M. Y. Torgov, B. A. Mendelsohn, C. G. Cerveny, D. F. Chace, R. L. DeBlanc, R. P. Gearing, T. D. Bovee, C. B. Siegall, J. A. Francisco, A. F. Wahl, D. L. Meyer and P. D. Senter,
Nat. Biotechnol., 2003, 21, 778; (b) G. Badescu, P. Bryant, M. Bird, K. Henseleit, J. Swierkosz, V. Parekh, R. Tommasi, E. Pawlisz, K. Jurlewicz, M. Farys, N. Camper, X. Sheng, M. Fisher, R. Grygorash, A. Kyle, A. Abhilash, M. Frigerio, J. Edwards and A. Godwin, Bioconjugate Chem., 2014, 25, 1124.

11 B.-Q. Shen, K. Xu, L. Liu and H. Raab, et al., Nat. Biotechnol., 2012, 30, 184. 